\title{
The block structure condition for symmetric hyperbolic systems
}

\author{
GuY MÉTIVIER \\ IRMAR Université de Rennes I \\ 35042 Rennes Cedex, France
}

\begin{abstract}
In the analysis of hyperbolic boundary value problems, the construction of Kreiss' symmetrizers relies on a suitable block structure decomposition of the symbol of the system. In this paper, we show that this block structure condition is satisfied by all symmetrizable hyperbolic systems of constant multiplicity.
\end{abstract}

In [2], H.O.Kreiss proved a maximal $L^{2}$ energy estimate for the solutions of mixed boundary-initial value problems for strictly hyperbolic systems and boundary conditions which satisfy the uniform Lopatinski condition (see also [8] for systems with complex coefficients). The proof is based on the construction of a symmetrizer. Thanks to the pseudodifferential calculus, the proof is reduced to the construction of an algebraic symmetrizer for the symbol of the equation (see e.g. [1]). The result extends to the case where the coefficients have finite smoothness ([3]) and, using the paradifferential calculus of J.M.Bony-Y.Meyer, to Lipschitzean coefficients ([7],[6]). Kreiss' analysis is extended to a class of characteristic boundary value problems in [5].

However, many interesting physical examples of hyperbolic systems are not strictly hyperbolic. For instance, Euler's equations of gas dynamics, Maxwell's equations or the equations of elasticity are not strictly hyperbolic. In the construction of Kreiss' symmetrizer, the strict hyperbolicity assumption is used at only one place, to prove that the symbol of the system has a suitable block decomposition near glancing modes (see Lemmas 2.5, 2.6 and 2.7 in [2]). In [5] and [3], it is shown that this block structure condition is satisfied by several nonstrictly hyperbolic systems such as the linearized shock front equations of gas dynamics ([3]), Maxwell's equations or the linearized shallow water equations ([5]). However, due to the lack of a simple criterion, one had to check the condition for each system separately.

The aim of this paper is to prove that the block structure assumption is satisfied for a large class of systems of physical interest which contains the examples above : the class of symmetrizable hyperbolic systems of constant multiplicity.

As a corollary, continuing the analysis as in [2], [1], [5] or [3], this implies the local well posedness of boundary value problems for linear symmetric (or 
symmetrizable) hyperbolic systems of constant multiplicity which satisfy the uniform Lopatinski condition. This result can be extended to nonlinear problems as in [4], [7], [6].

\section{The block structure Assumption}

Consider the symbol of a first order quasilinear system

$$
L(a, \tau, \eta, \xi):=\tau I_{N}+\xi A(a)+\eta_{1} B_{1}(a)+\ldots+\eta_{n} B_{n}(a) .
$$

Here, $\left.\left(\xi, \eta_{1}, \ldots \eta_{n}, \tau\right)\right)$ are the space-time frequencies, dual to the space-time variables $\left(x, y_{1}, \ldots, y_{n}, t\right)$. With applications to boundary value problems in mind, the boundary is $\{x=0\}$. The parameter $a$ is $(x, y, t, u)$, where $u$ is the placeholder of the solution and is considered as an independent set of variables. The coefficients $A$ and $B_{j}$ are $N \times N$ matrices which are $C^{\infty}$ functions of $a \in \mathbb{R}^{M}$ and $I_{N}$ is the identity matrix of dimension $N$. For simplicity, we use the notation

$$
\eta B:=\eta_{1} B_{1}+\ldots+\eta_{n} B_{n} .
$$

Definition 1.1. $L$ is a symmetrizable hyperbolic system of constant multiplicity if for all $a \in \mathcal{U}$ and $(\eta, \xi) \in \mathbb{R}^{d} \backslash\{0\}$, all the eigenvalues of $\xi A(a)+\eta B(a)$ are real and semi-simple and, locally, their multiplicity is independent of $(a, \eta, \xi)$.

Recall that $L$ is hyperbolic when all the eigenvalues of $\xi A(a)+\eta(a) B$ are real. It is strictly hyperbolic when they are simple. The eigenvalues are real and semi-simple, if and only if for all $(a, \eta, \xi)$, there is a definite positive matrix $S(a, \eta, \xi)$ such that

$$
S(a, \eta, \xi)(\xi A(a)+\eta B(a)) \quad \text { is hermitian symmetric }
$$

In particular, this occurs for symmetric hyperbolic systems.

When $L$ is hyperbolic with eigenvalues of constant multiplicity, there are $C^{\infty}$ functions $\lambda_{j}(a, \eta, \xi)$ and fixed integers $\alpha_{j}$ such that

$$
\lambda_{1}<\lambda_{2}<\ldots, \quad \text { and } \quad \operatorname{det} L(a, \tau, \eta, \xi)=\prod\left(\tau+\lambda_{j}(a, \eta, \xi)\right)^{\alpha_{j}}
$$

If in addition, the eigenvalues are semi-simple then the eigenprojectors $\Pi_{j}(a, \eta, \xi)$ are $C^{\infty}$ functions of $(a, \eta, \xi) \in \mathcal{U} \times\left(\mathbb{R}^{n+1} \backslash\{0\}\right)$ and there is a $C^{\infty}$ symmetrizer $S(a, \eta, \xi)$ such that $(1.2)$ holds. In this case the linear and nonlinear Cauchy problems are well posed.

We now assume that the boundary is not characteristic, i.e. that

$$
\forall a \in \mathcal{U}, \quad \operatorname{det} A(a) \neq 0 .
$$

The case of characteristic boundaries is discussed in section 3. In the study of boundary value problems, one introduces complex values of $\tau$ and $\xi$, and the matrices

$$
M(a, \tau, \eta, \gamma):=A(a)^{-1}\left((\tau-i \gamma) I_{N}+\eta B(a)\right) .
$$


We denote by $z=(a, \tau, \eta, \gamma)$ the variables in $\mathcal{U} \times \mathbb{R}^{n+2}$. $\Sigma$ denotes the set of $(\tau, \eta, \gamma) \in \mathbb{R}^{n+2}$ such that $\tau^{2}+|\eta|^{2}+\gamma^{2}=1$ and $\gamma \geq 0$ and $\Sigma_{0}$ is the subset of $(\tau, \eta, \gamma) \in \Sigma$ such that $\gamma=0$.

Definition 1.2 (The block structure condition). For all $\underline{z} \in \mathcal{U} \times \Sigma$, there is a neighbourhood $\mathcal{O}$ of $\underline{z}$ in $\mathcal{U} \times \mathbb{R}^{n+2}$ and there are matrices $T(z), C^{\infty}$ on $\mathcal{O}$ such that $T(z)^{-1} M(z) T(z)$ has the following block diagonal structure

$$
T(z)^{-1} M(z) T(z)=\left[\begin{array}{ccc}
Q_{1}(z) & \cdots & 0 \\
\vdots & \ddots & \vdots \\
0 & \cdots & Q_{\underline{p}}(z)
\end{array}\right]
$$

where the blocks $Q_{p}$ are $\nu_{p} \times \nu_{p}$ matrices which satisfy one of the following conditions :

i) $\operatorname{Im} Q_{p}(z):=\frac{1}{2 i}\left(Q_{p}-Q_{p}^{*}\right)$ is definite positive,

ii) $\operatorname{Im} Q_{p}(z)$ is definite negative,

iii) $\nu_{p}=1, Q_{p}(z)$ is real when when $\gamma=0$, and $\partial_{\gamma} Q_{p}(\underline{z}) \neq 0$,

iv) $\nu_{p}>1, Q_{p}(z)$ has real coefficients when $\gamma=0$, there is $\zeta_{p} \in \mathbb{R}$ such that

$$
Q_{p}(\underline{z})=\left[\begin{array}{cccc}
\zeta_{p} & 1 & 0 & \\
0 & \zeta_{p} & \ddots & 0 \\
& \ddots & \ddots & 1 \\
& & \cdots & \zeta_{p}
\end{array}\right]
$$

and the lower left hand corner of $\partial_{\gamma} Q_{p}(\underline{z})$ does not vanish.

In [2], O.Kreiss proved that the block structure condition is satisfied for strictly hyperbolic systems $L$. The main goal of this paper is to prove the following result.

Theorem 1.3. Suppose that $L$ is a symmetrizable hyperbolic system of constant multiplicity such that the boundary matrix $A$ is not characteristic. Then the block structure condition is satisfied.

This result can be extended to characteristic boundaries (see $\S 3$ ) and to higher order systems (see $\S 4$ below). All these results follow from a general statement, which we now explain.

Suppose that $M(z)$ is a $N \times N$ matrix, defined and $C^{\infty}$ for $z$ in a neighbourhood $\mathcal{O}$ of $\underline{z} \in \mathcal{U} \times \Sigma$. Introduce the notation $\Delta(z, \xi):=\operatorname{det}\left(\xi I_{N}+M(z)\right)$.

Assumption 1.4. i) When $\gamma \neq 0$, then $\Delta(\underline{z}, \xi) \neq 0$ for all $\xi \in \mathbb{R}$.

ii) When $\underline{z} \in \mathcal{U} \times \Sigma_{0}$, for all $\xi \in \mathbb{R}$ such that $\Delta(\underline{z}, \xi)=0$, there are a positive integer $\alpha$ and $C^{\infty}$ functions $\lambda(\bar{a}, \eta, \xi)$ and $\left.e(z, \xi)\right]$, defined on neighbourhoods 
of $(\underline{a}, \underline{\eta}, \underline{\xi})$ in $\mathcal{U} \times \mathbb{R}^{d-1} \times \mathbb{C}$ and $(\underline{z}, \underline{\xi})$ in $\mathcal{O} \times \mathbb{C}$ respectively, holomorphic in $\xi$ and such that

$$
\Delta(z, \xi)=e(z, \xi)(\tau-i \gamma+\lambda(a, \eta, \xi))^{\alpha}
$$

Moreover, $\lambda$ is real when $\xi$ is real and $e(\underline{z}, \underline{\xi}) \neq 0$.

In addition, there is a $C^{\infty}$ matrix valued function $\Pi(a, \eta, \xi)$ on a neighborhood of $(\underline{a}, \underline{\eta}, \underline{\xi})$, holomorphic in $\xi$, such that $\Pi$ is a projector of rank $\alpha$ and

$$
\operatorname{ker}(M(z)+\xi I d)=\Pi(a, \eta, \xi) \mathbb{C}^{N} \quad \text { when } \tau-i \gamma+\lambda(a, \eta, \xi)=0
$$

When $M$ is the matrix defined in (1.5), condition $i$ ) holds if and only if $L$ hyperbolic. If $\underline{z} \in \Sigma_{0}$ and $\underline{\xi}$ satisfy $\Delta(\underline{z}, \underline{\xi})=0$, then $(\underline{\eta}, \underline{\xi}) \neq 0$ since $(\underline{\tau}, \underline{\eta}) \neq 0$. Thus, by (1.3) there is a unique eigenvalue $\lambda_{j}$ such that $\underline{\tau}+\lambda_{j}(\underline{a}, \bar{\eta}, \bar{\xi})=0$. If $L$ is a symmetrizable hyperbolic system of constant multiplicity, $\bar{\lambda}_{j}$ and the eigenprojector $\Pi_{j}$ extend to a complex neighbourhood of $\underline{\xi}$ where (1.8) (1.9) are satisfied. Therefore, Theorem 1.3 follows from the next result.

Theorem 1.5. Suppose that the matrix $M(z)$ satisfies Assumption 1.4. Then $M$ satisfies the the block structure condition on a neighbourhood of $\underline{z}$.

\section{Proof of Theorem 1.5}

Let $\underline{\mu}_{k}$ be an eigenvalue of $\underline{M}:=M(\underline{z})$. We denote by $\beta_{k}$ its algebraic multiplicity and by $\underline{\mathbb{K}}_{k}$ the associated generalized eigenspace. There are $r>0$ and a neighbourhood $\mathcal{O}$ of $z$ such that for all $z \in \mathcal{O}$, the matrix $M(z)$ has exactly $\beta_{k}$ eigenvalues counted with their multiplicities, in $\left\{\left|\mu-\underline{\mu}_{k}\right|<r / 2\right\}$ and no eigenvalue in the annulus $\left\{r / 2 \leq\left|\mu-\underline{\mu}_{k}\right|<2 r\right\}$. The sum of the generalized eigenspaces associated to these eigenvalues is an invariant space of dimension $\beta_{k}$, which we denote by $\mathbb{K}_{k}(z)$. The spectral projector on $\mathbb{K}_{k}(z)$ is

$$
P_{k}(z)=\frac{1}{2 i \pi} \int_{\left|\mu-\underline{\mu}_{k}\right|=r}(M(z)-\mu I d)^{-1} d \mu .
$$

It is a $C^{\infty}$ function of $z \in \mathcal{O}$. Let $M_{k}(z)$ denote the restriction of $M(z)$ to $\mathbb{K}_{k}(z)$. Shrinking $\mathcal{O}$ if necessary, this construction can be performed for all the eigenvalues $\mu_{k}, k \in\{1, \ldots, \underline{k}\}$. The block structure condition is satisfied, if and only if there are bases of $\mathbb{K}_{k}(z)$, which are $C^{\infty}$ functions of $z$, such that for all $k$, the matrix of $M_{k}(z)$ in the corresponding basis of $\mathbb{K}_{k}(z)$ has the block diagonal form (1.6), where the blocks $Q_{p}$ satisfy one of the condition listed in Definition 1.2.

Consider a block $M_{k}$. There are two different cases, according as $\underline{\mu}_{k}$ is real or not.

Lemma 2.1. Suppose that $\operatorname{Im} \mu_{k} \neq 0$. Then there is a $C^{\infty}$ basis of $\mathbb{K}(z)$ such that the matrix of $M_{k}(z)$ in this basis has an imaginary part $\operatorname{Im} M_{k}$ which is either definite positive or definite negative. 
Proof. (see [2]) One can assume that $M_{k}(\underline{z})$ has a Jordan form. Changing the basis $\left(e_{1}, \ldots, e_{\beta_{k}}\right)$ of $\mathbb{K}_{k}(\underline{z})$ to $\left(e_{1}, \varepsilon e_{2}, \ldots, \varepsilon^{\beta_{k}-1} e_{\beta_{k}}\right)$, we obtain that

$$
M_{k}(\underline{z})=\underline{\mu}_{k} I_{\beta_{k}}+\left[\begin{array}{cccc}
0 & c_{1} & 0 & \cdots \\
0 & \ddots & \ddots & 0 \\
\vdots & \cdots & 0 & c_{\beta_{k}-1} \\
0 & \cdots & & 0
\end{array}\right]
$$

where $c_{l} \in\{0, \varepsilon\}$. If $\varepsilon$ is small, $\operatorname{Im} M_{k}(z)$ is positive definite [resp. negative definite] at $z=\underline{z}$ when $\operatorname{Im} \underline{\mu}_{k}>0$ [resp. $\operatorname{Im} \underline{\mu}_{k}<0$ ]. By continuity, this property remains satisfied for $z$ close to $\underline{z}$.

Consider next a block $M_{k}$ with $\mu_{k} \in \mathbb{R}$. Note that Assumption 1.4, implies that necessarily $\underline{\gamma}=0$.

Proposition 2.2. Suppose that $\underline{\gamma}=0$. Consider a real eigenvalue $\mu_{k}$ of $M(\underline{z})$. Then there is a $C^{\infty}$ basis of $\mathbb{K}_{k}(\bar{z})$ such that the matrix of $M_{k}(z)$ has the block structure (1.6) where the blocks $Q_{p}(z)$ satisfy either the condition iii) or the condition iv) of Definition 1.2.

Introduce the notation $\underline{\xi}=-\underline{\mu}_{k}$. Then, $\Delta(\underline{z}, \underline{\xi})=0$, and (1.8) (1.9) hold. In the strictly hyperbolic case, i.e. when $\lambda$ is a simple eigenvalue and $\alpha=1$ in (1.8), $M_{k}$ contains one block $Q_{p}$. In the general case, there are $\alpha$ blocks, and the main difficulty is to prove that $M_{k}$ has a smooth decomposition (1.6).

First we study the structure of the characteristic polynomial of $M_{k}(z)$.

Lemma 2.3. There is a neighbourhood $\mathcal{O}$ of $\underline{z}$ and there is a monic polynomial in $\xi, D(z, \xi)$, with $C^{\infty}$ coefficients in $z \in \mathcal{O}$ such that for all $z \in \mathcal{O}$,

$$
\Delta_{k}(z, \xi):=\operatorname{det}\left(\xi I_{\mathbb{K}_{k}(z)}+M_{k}(z)\right)=(D(z, \xi))^{\alpha}
$$

In particular, $\beta_{k}=\nu \alpha$ where $\nu$ is the degree of $D$. The coefficients of $D$ are real when $\gamma=0$ and

$$
\frac{\partial D}{\partial \gamma}(\underline{z}, \underline{\xi}) \neq 0
$$

Furthermore, the set $\mathcal{O}^{*}$ of points $z \in \mathcal{O}$ such that $D(z, \cdot)$ has only simple roots is dense in $\mathcal{O}$.

Proof. In (2.1), $I_{\mathbb{K}_{k}(z)}$ denotes the indentity operator on $\mathbb{K}_{k}(z)$. One has

$$
\operatorname{det}(M(z)+\xi I d)=\Delta_{k}(z, \xi) e_{1}(z, \xi),
$$

where $e_{1}$ is the determinant of the restriction of $M(z)$ to the range of $I_{N}-P_{k}(z)$. Thus $e_{1}(\underline{z}, \xi) \neq 0$. With (1.8), this implies that, shrinking the neighbourhoods if necessary, there is an open disc $\mathcal{V}$ centered at $\underline{\xi}$ such that for $(z, \xi) \in \mathcal{O} \times \mathcal{V}$

$$
\Delta_{k}(z, \xi)=(\tau-i \gamma+\lambda(a, \eta, \xi))^{\alpha} e_{2}(z, \xi)
$$


where $e_{2}(z, \xi)$ is $C^{\infty}$ in $z$, holomorphic in $\xi$ and does not vanish on $\mathcal{O} \times \mathcal{V}$. Moreover, for all $z \in \mathcal{O}$, all the roots of $\Delta_{k}(z, \cdot)$ belong to $\mathcal{V}$.

Since $\Delta_{k}$ is a polynomial in $\xi,(2.3)$ implies that $\underline{\xi}$ is a root of finite order of $\underline{\tau}+\lambda(\underline{a}, \underline{\eta}, \xi)=0$. Thus there is $\nu \geq 1$ such that

$$
\frac{\partial^{l} \lambda_{j}}{\partial \xi^{l}}(\underline{a}, \underline{\eta}, \underline{\xi})=0 \quad \text { for } l=1, \ldots, \nu-1 \quad \text { and } \quad \frac{\partial^{\nu} \lambda_{j}}{\partial \xi^{\nu}}(\underline{a}, \underline{\eta}, \underline{\xi}) \neq 0
$$

We are in position to apply Weierstrass preparation theorem. There is a monic polynomial of degree $\nu$ in $\xi, D(z, \xi)$, with $C^{\infty}$ coefficients in $z$, and there is a nonvanishing function $e_{3}(z, \xi)$ defined for $(z, \xi)$ in a neighbourhood of $(\underline{z}, \underline{\xi})$, holomorphic in $\xi, C^{\infty}$ in $z$ and such that

$$
\tau-i \gamma+\lambda_{j}(a, \eta, \xi)=e_{3}(z, \xi) D(z, \xi), \quad D(\underline{z}, \xi)=(\xi-\underline{\xi})^{\nu} .
$$

The usual version of Weierstrass preparation theorem also assumes analyticity in the parameters. Since the coefficients are only $C^{\infty}$, and the Malgrange preparation theorem only provides (2.5) for real $\xi$, we include a short proof based on the known explicit expressions of $D$ and $e_{3}$. Let $f(z, \xi)$ denote the left hand side of (2.5). There are $r>0$ and a neighbourhood $\mathcal{O}$ of $\underline{z}$ such that for all $z \in \mathcal{O}$, there is a unique decomposition

$$
f(z, \xi)=p(z, \xi) e^{h(z, \xi)}
$$

where $p$ is a polynomial of degree $\nu$ with $\nu$ roots in the disc $\{|\xi-\xi|<r / 2\}$ and $h$ is holomorphic in $\{|\xi-\underline{\xi}|<2 r\}$ such that $h(z, \underline{\xi})=0$. For $|\xi-\underline{\xi}|<r$, one has

$$
\partial_{\xi} h(z, \xi)=\frac{1}{2 i \pi} \int_{|w-\underline{\xi}|=r} \frac{\partial_{\xi} f(z, w)}{f(z, w)} \frac{d w}{w-\xi} .
$$

This shows that $\partial_{\xi} h$ and therefore $h$ and $p=f e^{-h}$ are $C^{\infty}$ in $z$. Factoring out the coefficient of $\xi^{\nu}$ implies (2.5).

The Schwarz reflection principle implies that $\lambda(a, \eta, \bar{\xi})=\overline{\lambda(a, \eta, \xi)}$. Thus the explicit formula above shows that when $\gamma=0, D$ has real coefficients.

Combining (2.3) and (2.5) implies that $\Delta_{k}=D^{\alpha} e_{4}$ on a neighbourhood $\mathcal{O} \times \mathcal{V}$ of $(\underline{z}, \underline{\xi})$ where $e_{4}$ does not vanish. Moreover, shrinking $\mathcal{O}$ if necessary, all the roots of the polynomials $\Delta_{k}(z, \cdot)$ and $D(z, \cdot)$ belong to $\mathcal{V}$. Thus $e_{4}(z, \cdot)$ is constant and, since both polynomials $D(z, \cdot)$ and $\Delta_{k}(z, \cdot)$ are monic, $(2.1)$ follows.

The property (2.2) immediately follows from (2.5). Shrinking the neighbourhoods, one can assume that $\partial_{\gamma} D$ does not vanish on $\mathcal{O} \times \mathcal{V}$. Suppose that $z_{1}=\left(a_{1}, \tau_{1}, \eta_{1}, \gamma_{1}\right) \in \mathcal{O}$ and $D\left(z_{1}, \cdot\right)$ has a root of multiplicity $\nu_{1}$ at $\xi_{1}$. Since $\partial_{\gamma} D$ does not vanish, for $z=\left(a_{1}, \tau_{1}, \eta_{1}, \gamma_{1}+s\right) \in \mathcal{O}$, one has

$$
D(z, \xi)=c_{1}\left(\xi-\xi_{1}\right)^{\nu_{1}}+O\left(\xi-\xi_{1}\right)^{\nu_{1}+1}+c_{2} s+O\left(s^{2}\right)
$$

with $c_{1} \neq 0$ and $c_{2} \neq 0$. Thus, for $s$ small enough, the multiple root splits into simple roots. This proves that $\mathcal{O}^{*}$ is dense in $\mathcal{O}$. 
Corollary 2.4. Suppose that $\underline{\xi}$ is a simple root of $\underline{\tau}+\lambda(\underline{a}, \underline{\eta}, \cdot)=0$. Then, there is a $C^{\infty}$ function $\mu_{k}(z)$ on a neighbourhood of $\underline{z}$ such that $M_{k}(z)=\mu_{k} I_{\mathbb{K}_{k}(z)}$. Moreover, $\mu_{k}$ is real when $\gamma=0$ and $\partial_{\gamma} \mu_{k}(\underline{z}) \neq 0$. In particular $M_{k}$ has the form (1.6), with one dimensional blocks $Q_{p}$ equal to $\mu_{k}(z)$. They satisfy condition iii) of Definition 1.2.

Proof. When $\nu=1$, there is a $C^{\infty}$ function $\mu_{k}(z)$ such that $D(z, \xi)=\xi+\mu_{k}(z)$. Thus, (1.8) implies that $-\mu_{k}(z)$ is an eigenvalue of constant multiplicity $\alpha$ of $M(z)$. Moreover, (1.9) and (2.5) imply that

$$
\left(M(z)-\mu_{k}(z)\right) \Pi\left(a, \eta,-\mu_{k}(z)\right)=0 .
$$

Since the rank of $\Pi$ is equal to $\alpha, \mu_{k}(z)$ is a semi-simple eigenvalue of $M(z)$. The properties of $\mu_{k}$ follow from Lemma 2.1

It remains to study the most difficult case, when the degree $\nu$ of $D$ is larger than one. In the strictly hyperbolic case, this means that $\underline{\xi}$ is a multiple root of $\Delta(\underline{z}, \underline{\xi})=0$, which means that $(\underline{z}, \underline{\xi})$ is a "glancing" mode.

We now assume that $\nu>1$. First, we study the structure of $M_{k}(\underline{z})$.

Lemma 2.5. The operators $\underline{P}_{l}=\left(\partial_{\xi}^{l} \Pi\right)(\underline{a}, \underline{\eta}, \underline{\xi})$ satisfy

$$
\left(\underline{M}-\underline{\mu}_{k}\right) \underline{P}_{0}=0 \quad \text { and } \quad\left(\underline{M}-\underline{\mu}_{k}\right) \underline{P}_{l}=-l \underline{P}_{l-1} \quad \text { for } \quad l=1, \ldots \nu-1
$$

Moreover, the generalized eigenspace of $\underline{M}$ associated to $\underline{\mu}_{k}$ is the direct sum

$$
\underline{\mathbb{K}}_{k}=\bigoplus_{l=0}^{\nu-1} \underline{P}_{l} \underline{\mathbb{E}}_{0}, \quad \underline{\mathbb{E}}_{0}:=\underline{P}_{0} \mathbb{C}^{N}
$$

Proof. By (2.4), $\lambda(\underline{a}, \underline{\eta}, \xi)+\underline{\tau}=O(\xi-\underline{\xi})^{\nu}$. Introduce the notation $z(\xi)=$ $(\underline{a}, \tau, \underline{\eta}, \gamma)$ with $\tau-i \gamma=-\lambda(\underline{a}, \underline{\eta}, \xi)$. By $(\overline{1} .9)$, one has

$$
\left(\underline{M}+\xi I_{N}\right) \Pi(\underline{a}, \underline{\eta}, \xi)=M(\underline{z})-M(z(\xi))=O(|\tau-\underline{\tau}|+|\gamma|)=O\left((\xi-\underline{\xi})^{\nu}\right)
$$

and (2.7) follows. Introduce $\underline{\mathbb{E}}_{0}:=\underline{P}_{0} \mathbb{C}^{N}$ and for $l=1, \ldots, \nu-1, \underline{\mathbb{E}}_{l}:=\underline{P}_{l} \underline{\mathbb{E}}_{0}$. Then (2.7) implies that

$$
\left(\underline{M}-\underline{\mu}_{k}\right) \underline{\mathbb{E}}_{0}=0, \quad\left(\underline{M}-\underline{\mu}_{k}\right) \underline{\mathbb{E}}_{l}=\underline{\mathbb{E}}_{l-1}
$$

By definition, $\operatorname{dim} \underline{\mathbb{E}}_{0}=\operatorname{rank} \underline{\Pi}=\alpha$ and $\operatorname{dim} \underline{\mathbb{E}}_{l} \leq \operatorname{dim} \underline{\mathbb{E}}_{0}$. On the other hand, (2.8) implies that for $l \geq 1, \operatorname{dim} \underline{\mathbb{E}}_{l} \geq \operatorname{dim} \underline{\mathbb{E}}_{l-1}$. Therefore

$$
\forall l \in\{0, \ldots, \nu-1\}, \quad \operatorname{dim} \underline{\mathbb{E}}_{l}=\alpha
$$

Consider $\left(u_{0}, \ldots, u_{\nu-1}\right) \in \mathbb{E}_{0}^{\nu}$. Suppose that $\sum \underline{P}_{l} u_{l}=0$. Applying $\left(\underline{M}-\underline{\mu}_{k}\right)^{\nu-1}$ to this equation, implies that $0=\left(\underline{M}-\underline{\mu}_{k}\right)^{\nu-1} \underline{P}_{\nu-1} u_{\nu-1}=\underline{P}_{0} u_{\nu-1}=u_{\nu-1}$. Inductively, one shows that all the $u_{l}$ vanish. This proves that the sum $\underline{\mathbb{E}}:=$ $\underline{\mathbb{E}}_{0} \oplus \ldots \oplus \mathbb{E}_{\nu-1}$ is direct. In particular, $\operatorname{dim} \underline{\mathbb{E}}=\nu \alpha=\beta_{k}$.

By $(2.8),\left(\underline{M}-\mu_{k}\right)^{\nu} \underline{\mathbb{E}}=0$, thus $\underline{\mathbb{E}}$ is contained in the generalized eigenspace $\underline{\mathbb{K}}_{k}$, which is also of dimension $\beta_{k}$. Therefore $\underline{\mathbb{E}}=\underline{\mathbb{K}}_{k}$. 
Let $\left(\underline{e}_{0,1}, \ldots, \underline{e}_{0, \alpha}\right)$ be a basis of $\underline{\mathbb{E}}_{0}$. For $l=1, \ldots, \nu-1$, and $p=1, \ldots, \alpha$, introduce

$$
\underline{e}_{l, p}=\frac{(-1)^{l}}{l !} \underline{P}_{l} \underline{e}_{0, p} .
$$

Then the $\left\{\underline{e}_{l, p}\right\}$ form a basis of $\underline{\mathbb{K}}_{k}$. We denote by $\underline{\mathbb{F}}_{p}$ the space generated by $\left(\underline{e}_{0, p}, \ldots, \underline{e}_{\nu-1, p}\right)$. Thus

$$
\underline{\mathbb{K}}_{k}=\underline{\mathbb{F}}_{1} \oplus \ldots \oplus \underline{\mathbb{F}}_{\alpha} .
$$

In this block decomposition of the generalized eigenspace, (2.7) implies that the matrix of $\underline{M}_{k}$ in the bases above has the following diagonal block structure

$$
\underline{M}_{k}=\left[\begin{array}{ccc}
\underline{Q} & \cdots & 0 \\
\vdots & \ddots & \vdots \\
0 & \cdots & \underline{Q}
\end{array}\right]
$$

where $\underline{Q}$ is the $\nu \times \nu$ matrix (1.7) with $\zeta_{p}=\underline{\mu}_{k}$.

Our goal is to extend the splitting (2.11) when $z$ is close to $\underline{z}$, so that the $\mathbb{F}_{p}(z)$ are invariant by $M(z)$ and depend smoothly on $z$. Recall that $\Pi(a, \eta, \xi)$ is defined for $\xi$ in a complex disc $\{|\xi-\xi|<2 r\}$. Shrinking $\mathcal{O}$ if necessary, we can assume that for all $z \in \mathcal{O}$, the $\nu$ complex roots of $D(z, \cdot)$ satisfy $\left|\xi_{p}-\underline{\xi}\right| \leq r / 2$. Therefore, we can define for $z \in \mathcal{O}$ and $l \in\{0, \ldots, \nu-1\}$,

$$
P_{l}(z):=\frac{l !(\nu-l-1) !}{2 i \pi \nu !} \int_{|\xi-\underline{\xi}|=r} \Pi(a, \eta, \xi) \frac{\partial_{\xi}^{l+1} D(z, \xi)}{D(z, \xi)} d \xi .
$$

They are $C^{\infty}$ functions on $\mathcal{O}$. Since $D(\underline{z}, \xi)=(\xi-\underline{\xi})^{\nu}$, Cauchy's formula implies that

$$
P_{l}(\underline{z})=\left(\partial_{\xi}^{l} \Pi\right)(\underline{a}, \underline{\eta}, \underline{\xi})=\underline{P}_{l} .
$$

Moreover, when $z \in \mathcal{O}^{*}$, the roots $\left(\xi_{1}, \ldots, \xi_{\nu}\right)$ of $D(z, \cdot)$ are simple and

$$
P_{l}(z)=\sum_{m=1}^{\nu} c_{l}\left(z, \xi_{m}\right) \Pi\left(a, \eta, \xi_{m}\right)
$$

where

$$
c_{l}\left(z, \xi_{m}\right)=\frac{l !(\nu-l-1) !}{\nu !} \frac{\partial_{\xi}^{l+1} D\left(z, \xi_{m}\right)}{\partial_{\xi} D\left(z, \xi_{m}\right)} \in \mathbb{C} .
$$

Recall that $\left\{\underline{e}_{0, p}\right\}_{1 \leq p \leq \alpha}$ is a basis of $\underline{\mathbb{E}}_{0}$. For $l \in\{0, \ldots, \nu-1\}$ and $p \in$ $\{1, \ldots, \alpha\}$, consider

$$
e_{l, p}(z):=\frac{(-1)^{l}}{l !} P_{l}(z) \underline{e}_{0, p}
$$

and $\mathbb{F}_{p}(z)$ the linear space spanned by $\left(e_{0, p}(z), \ldots, e_{\nu-1, p}(z)\right)$.

Lemma 2.6. Shrinking $\mathcal{O}$ if necessary, for $z \in \mathcal{O}$, the vectors $\left\{e_{l, p}(z)\right\}$ are linearly independent. Their span $\mathbb{F}_{1}(z) \oplus \ldots \oplus \mathbb{F}_{\alpha}(z)$ is equal to the space $\mathbb{K}_{k}(z)$. Moreover, for all $p, \mathbb{F}_{p}(z)$ is invariant by $M(z)$ and the matrix of $M(z)_{\mathbb{F}_{p}(z)}$ in the basis $\left\{e_{l, p}(z)\right\}_{0 \leq l \leq \nu-1}$ is independent of $p$. 
Proof. (2.14) implies that $e_{l, p}(\underline{z})=\underline{e}_{l, p}$. Therefore, for $z$ close to $\underline{z}$, the vectors $e_{l, p}(z)$ are linearly independent.

Suppose that $z \in \mathcal{O}^{*}$. Lemma 2.1 implies that $M(z)$ has $\nu$ pairwise different eigenvalues, $\left(-\xi_{1}, \ldots,-\xi_{\nu}\right)$ such that $D\left(z, \xi_{m}\right)=0$. By $(2.5)$, they satisfy $\tau-i \gamma+\lambda\left(a, \eta, \xi_{m}\right)=0$. Therefore (1.9) implies that the kernel $\mathbb{L}_{m}(z)$ of $M(z)+\xi_{m} I_{N}$ is the range of $\Pi\left(a, \eta, \xi_{m}\right)$ and

$$
M(z) \Pi\left(a, \eta, \xi_{m}\right)=-\xi_{m} \Pi\left(a, \eta, \xi_{m}\right) .
$$

In particular, the dimension of $\mathbb{L}_{m}(z)$ is $\alpha$. Since the $\xi_{m}$ are pairwise distinct the spaces $\mathbb{L}_{m}(z)$ are in direct sum. Because, $\beta_{k}=\nu \alpha$, this implies that the eigenvalues $-\xi_{m}$ of $M(z)$ are semi-simple and that $\mathbb{L}_{1}(z) \oplus \ldots \oplus \mathbb{L}_{\nu}(z)=\mathbb{K}_{k}(z)$.

For $(z, \xi)$ close to $(\underline{z}, \underline{\xi}), \Pi(a, \eta, \xi)$ is close to $\Pi(\underline{a}, \underline{\eta}, \underline{\xi})$ and

$$
\widetilde{e}_{p}(a, \eta, \xi):=\frac{(-1)^{l}}{l !} \Pi(a, \eta, \xi) \underline{e}_{0, p}, \quad 1 \leq p \leq \alpha
$$

form a basis of $\Pi(a, \eta, \xi) \mathbb{C}^{N}$. In particular, $\left\{\widetilde{e}_{p}\left(a, \eta, \xi_{m}\right\}_{1 \leq p \leq \alpha}\right.$ is a basis of $\mathbb{L}_{m}(z)$. Since the $\mathbb{L}_{m}$ are in direct sum the $\left\{\widetilde{e}_{p}\left(a, \eta, \xi_{m}\right)\right\}_{p, m}$ are linearly independent and form a basis of $\mathbb{K}_{k}(z)$. (2.15) implies that for all $l$ and $p$,

$$
e_{l, p}(z)=\sum_{m=1}^{\nu} c_{l}\left(z, \xi_{m}\right) \widetilde{e}_{p}\left(a, h, \xi_{m}\right)
$$

Let $\widetilde{\mathbb{F}}_{p}(z)$ denote the space spanned by $\left\{\widetilde{e}_{p}\left(z, \xi_{m}\right)\right\}_{1 \leq m \leq \nu}$. Then (2.18) implies that $\mathbb{F}_{p}(z) \subset \widetilde{\mathbb{F}}_{p}(z)$. Since they have the same dimension, they are equal and

$$
\begin{aligned}
\mathbb{F}_{1}(z) \oplus \ldots \oplus \mathbb{F}_{\alpha}(z) & =\widetilde{\mathbb{F}}_{1}(z) \oplus \ldots \oplus \widetilde{\mathbb{F}}_{\alpha}(z) \\
& =\mathbb{L}_{1}(z) \oplus \ldots \oplus \mathbb{L}_{\nu}(z)=\mathbb{K}_{k}(z) .
\end{aligned}
$$

In addition, (2.17) implies that $\widetilde{\mathbb{F}}_{p}(z)$ and hence $\mathbb{F}_{p}(z)$ is invariant by $M(z)$. The matrix of $M(z)_{\mid \mathbb{F}_{p}(z)}$ in the basis $\left\{\widetilde{e}_{p}\left(z, \xi_{m}\right)\right\}_{1 \leq m \leq \nu}$ is diagonal with entries $\left\{-\xi_{m}\right\}$ independent of $p$. Since the coefficients $c_{l}\left(z, \xi_{m}\right)$ in (2.18) are also independent of $p$, it follows that the matrix of $M(z)_{\mid \mathbb{F}_{p}(z)}$ in the basis $\left\{e_{l, p}(z)\right\}_{0 \leq l \leq \nu-1}$ is independent of $p$.

Because $\mathcal{O}^{*}$ is dense in $\mathcal{O}$ and the $e_{l, p}(z)$ are smooth and linearly independent for $z \in \mathcal{O}$, it remains true that for all $z \in \mathcal{O}, \mathbb{F}_{p}(z)$ is invariant by $M(z), \mathbb{K}_{k}(z)=$ $\mathbb{F}_{1}(z) \oplus \ldots \oplus \mathbb{F}_{\alpha}(z)$ and the matrix of $M(z)_{\mid \mathbb{F}_{p}(z)}$ in the basis $\left\{e_{l, p}(z)\right\}_{0 \leq l \leq \nu-1}$ is independent of $p$.

In the block decomposition $\mathbb{K}_{k}(z)=\mathbb{F}_{1}(z) \oplus \ldots \oplus \mathbb{F}_{\alpha}(z)$, the lemma implies that the matrix of $M_{k}(z)$ has the following diagonal block structure

$$
M_{k}(z)=\left[\begin{array}{ccc}
Q(z) & \ldots & 0 \\
\vdots & \ddots & \vdots \\
0 & \ldots & Q(z)
\end{array}\right]
$$


where $Q(z)$ is a smooth $\nu \times \nu$ matrix. Moreover, $Q(\underline{z})=\underline{Q}$ is the matrix (1.7) with $\zeta_{p}=\underline{\mu}_{k}$.

Knowing (2.19), the proof of Proposition 2.2 continues as in [8].

Lemma 2.7. Shrinking the neighbourhood $\mathcal{O}$ if necessary, there are bases in the spaces $\mathbb{F}_{p}(z)$ which are $C^{\infty}$ in $z \in \mathcal{O}$ and such that (2.19) holds, $Q(z)$ is real when $\gamma=0$ and the left lower hand corner entry of $\partial_{\gamma} Q(\underline{z})$ does not vanish.

Proof. Lemma 2.1 and (2.19) imply that

$$
(D(z, \xi))^{\alpha}=\Delta_{k}(z, \xi)=\operatorname{det}\left(M_{k}(z)+\xi I_{\beta_{k}}\right)=\left(\operatorname{det}\left(Q(z)+\xi I_{\nu}\right)\right)^{\alpha}
$$

Therefore the monic polynomials of degree $\nu \operatorname{det}\left(Q(z)+\xi I_{\nu}\right)$ and $D(z, \xi)$ are equal. Because $Q(\underline{z})$ has the form (1.7), it is shown in [8] that for $z$ sufficiently close to $\underline{z}$, there is a smooth transformation $T(z)$ such that $T(\underline{z})=I_{\nu}$ and

$$
\widetilde{Q}(z):=T(z)^{-1} Q(z) T(z)=\underline{Q}+\left[\begin{array}{cccc}
q_{1}(z) & 0 & \cdots & 0 \\
\vdots & \vdots & \ddots & \vdots \\
q_{\nu}(z) & 0 & \ldots & 0
\end{array}\right] .
$$

Thus, (2.20) implies that

$$
D(z, \xi)=\operatorname{det}\left(\widetilde{Q}(z)+\xi I_{\nu}\right)=(\xi-\underline{\xi})^{\nu}+\sum_{l=1}^{\nu}(-1)^{l} q_{l}(z)(\xi-\underline{\xi})^{\nu-l}
$$

Since $D$ has real coefficients when $\gamma=0$, this implies that the $q_{l}(z)$ and therefore $\widetilde{Q}(z)$ are real when $\gamma=0$. In addition, Lemma 2.1 and (2.21) imply that

$$
\frac{\partial D}{\partial \gamma}(\underline{z}, \underline{\xi})=\frac{\partial q_{\nu}}{\partial \gamma}(\underline{z}) \neq 0 \text {. }
$$

Therefore $\widetilde{Q}(z)$ satisfies the property $i v$ ) of Definition 1.2 and the proofs of Proposition 2.2 and Theorem 1.5 are complete.

\section{Characteristic boundaries}

In [5], A.Majda and S.Osher have extended Kreiss' analysis to a class of characteristic boundary value problems. Our purpose here is to show that the block structure assumptions introduced in [5] are automatically satisfied by symmetrizable hyperbolic systems of constant multiplicity.

Consider a system (1.1). In place of (1.4) we now assume that the boundary matrix has a constant rank, i.e. that there is $N^{\prime} \leq N$ such that

$$
\forall a \in \mathcal{U}, \quad \operatorname{rank} A(a)=N^{\prime} .
$$


Thus, one can assume that

$$
A(a)=\left[\begin{array}{cc}
0 & 0 \\
0 & A_{22}(a)
\end{array}\right], \quad \operatorname{det} A_{22}(a) \neq 0 .
$$

We also introduce the notations $z=(a, \tau, \eta, \gamma)$ and

$$
G(z):=(\tau-i \gamma) I_{N}+\eta B(a)=\left[\begin{array}{ll}
G_{11}(z) & G_{12}(z) \\
G_{21}(z) & G_{22}(z)
\end{array}\right]
$$

The substitute for the matrix $M(1.5)$ is

$$
M^{\prime}(z):=A_{22}^{-1}\left(G_{22}-G_{21} G_{11}^{-1} G_{12}\right) .
$$

which is defined when $\operatorname{det} G_{11} \neq 0$.

Theorem 3.1. Suppose that $L$ is a symmetrizable hyperbolic system with constant multiplicity satisfying (3.1). Then, for all $\underline{z} \in \Sigma$ such that $\operatorname{det} G_{11}(\underline{z}) \neq 0$ the matrix $M^{\prime}(z)$ satisfies the block structure condition of Definition 1.2 on a neighbourhood of $\underline{z}$.

When $\operatorname{det} G_{11}$ tends to 0 , it is shown in [5] that $M^{\prime}(z)$ has a smooth block decomposition into a singular part and a regular part. The block structure assumptions of [5] also concern the regular part of $M^{\prime}(z)$ when $\operatorname{det} G_{11} \rightarrow 0$. We now give an equivalent presentation of these conditions.

Consider $\underline{z} \in \mathcal{U} \times \Sigma$ such that the polynomial $\Delta(\underline{z}, \xi):=\operatorname{det}(\xi A+G(\underline{z}))$ does not vanish identically. This, it has a finite number of roots, $\tilde{N} \leq N^{\prime}$. Fix $R>0$ such that the roots of $\Delta(\underline{z}, \cdot)$ are contained in the disc $\{|\xi|<R / 2\}$. There is a neighbourhood $\mathcal{O}$ of $\underline{z}$ such that for $z \in \mathcal{O}$, one has

$$
\Delta(z, \xi):=\operatorname{det}(\xi A+G(z))=\widetilde{\Delta}(z, \xi) e(z, \xi)
$$

where $\widetilde{\Delta}$ and $e$ are polynomials in $\xi$ with $C^{\infty}$ coefficients in $z$. $\widetilde{\Delta}$ is monic of degree $\tilde{N}$ and its roots are contained in $\{|\xi|<R / 2\}$. Moreover, $e(z, \cdot)$ does not vanish on $\{|\xi|<2 R\}$ and its roots tend to $\infty$ when $z$ tends to $\underline{z}$.

Lemma 3.2. There are invertible matrices $T_{1}(z)$ and $T_{2}(z)$ defined and $C^{\infty}$ on a neighbourhood $\mathcal{O}$ of $\underline{z}$, such that

$$
T_{2}(z)(\xi A+G(z)) T_{1}(z)=\left[\begin{array}{cc}
\xi \widetilde{A}(z)+\widetilde{G}(z) & 0 \\
0 & \xi A_{b}(z)+G_{b}(z)
\end{array}\right]
$$

where $\widetilde{A}(z), \widetilde{G}(z), A_{b}(z)$ and $G_{b}(z)$ are $C^{\infty}$ matrices on $\mathcal{O}$. Moreover, $\widetilde{A}(z)$ is invertible and $\widetilde{M}(z)=\widetilde{A}^{-1}(z) \widetilde{G}(z)$ satisfies

$$
\operatorname{det}\left(\xi I_{\tilde{N}}+\widetilde{M}(z)\right)=\widetilde{\Delta}(z, \xi) .
$$


Proof. By (3.4), $\xi A+G(z)$ is invertible for $R / 2<|\xi|<2 R$. Introduce the projectors

$$
\begin{aligned}
& P(z)=\frac{1}{2 i \pi} \int_{|\xi|=R}(G+\xi A)^{-1} A d \xi, \\
& P^{\prime}(z)=\frac{1}{2 i \pi} \int_{|\xi|=R} A(G+\xi A)^{-1} d \xi .
\end{aligned}
$$

They are $C^{\infty}$ functions of $z \in \mathcal{O}$. Denote by $\widetilde{\mathbb{K}}(z), \mathbb{K}_{b}(z), \widetilde{\mathbb{K}}^{\prime}(z)$ and $\mathbb{K}_{b}^{\prime}(z)$ the range of $P(z), I_{N}-P(z), P^{\prime}(z)$ and $I_{N}-P^{\prime}(z)$ respectively. One has

$$
P^{\prime}(z) A=A P(z), \quad P^{\prime}(z) G(z)=G(z) P(z) .
$$

This implies that $A$ and $G(z)$ map $\widetilde{\mathbb{K}}(z)$ [resp. $\left.\mathbb{K}_{b}(z)\right]$ into $\widetilde{\mathbb{K}}^{\prime}(z)$ [resp. $\left.\mathbb{K}_{b}^{\prime}(z)\right]$. Taking bases in $\widetilde{\mathbb{K}}(z), \mathbb{K}_{b}(z), \widetilde{\mathbb{K}}^{\prime}(z)$ and $\mathbb{K}_{b}^{\prime}(z)$ implies $(3.5)$.

The definition (3.7) shows that $P$ vanishes on ker $A$. Thus, if $A P u=0$ one has $P(P u)=0$, hence $P u=0$. This proves that $A$ is one to one from $\widetilde{\mathbb{K}}(z)$ to $\widetilde{\mathbb{K}}^{\prime}(z)$. Similarly, $P^{\prime}$ is valued in the range of $A$, thus for all $f \in \widetilde{\mathbb{K}}^{\prime}$, there is $u$ such that $f=A u$, thus $f=P^{\prime} f=P^{\prime} A u=A P u$, showing that $A$ maps $\widetilde{\mathbb{K}}(z)$ onto $\widetilde{\mathbb{K}}^{\prime}(z)$. Therefore, the matrix $\widetilde{A}(z)$ in (3.5) is invertible.

For $|\zeta| \neq R$, introduce

$$
U(z, \zeta)=\frac{1}{2 i \pi} \int_{\left|\xi-\underline{\xi}_{k}\right|=r}(G+\xi A)^{-1} \frac{d \xi}{\zeta-\xi} .
$$

Then

$$
(\zeta A+G(z)) U(z, \zeta)= \begin{cases}P^{\prime}(z) & \text { when }|\zeta|>R, \\ P^{\prime}(z)-I_{N} & \text { when }|\zeta|<R .\end{cases}
$$

Moreover, changing the path of integration in (3.7) (3.8), implies that

$$
\begin{cases}U(z, \zeta)=P(z) U(z, \zeta)=U(z, \zeta) P^{\prime}(z) & \text { when }|\zeta|>R \\ P(z) U(z, \zeta)=U(z, \zeta) P^{\prime}(z)=0 & \text { when }|\zeta|<R\end{cases}
$$

This implies that $(\zeta \widetilde{A}+\widetilde{G})\left[\right.$ resp. $\left.\left(\zeta A_{b}+G_{\mathrm{b}}\right)\right]$ is invertible when $|\zeta|>R$ [resp. $|\zeta|<R]$. We also know that both matrices are invertible for $R / 2<|\zeta|<R$. Therefore the roots of $\operatorname{det}\left(\zeta I_{\tilde{N}}+\widetilde{M}\right)\left[\operatorname{resp} \cdot \operatorname{det}\left(\zeta A_{b}+G_{b}\right)\right]$ are located in $|\zeta| \leq$ $R / 2[$ resp. $|\zeta| \geq 2 R]$. Since

$$
\Delta(z, \zeta)=\operatorname{det} T_{2} \operatorname{det} T_{1} \operatorname{det} \widetilde{A} \operatorname{det}\left(\zeta I_{\tilde{N}}+\widetilde{M}\right) \operatorname{det}\left(\zeta A_{b}+G_{\mathrm{b}}\right),
$$

(3.6) follows.

Theorem 3.3. Suppose that $L$ is a symmetrizable hyperbolic system with constant multiplicity such that the boundary matrix A satisfies (3.1). Consider $\underline{z} \in \Sigma$ such that the polynomial $\Delta(\underline{z}, \cdot)$ does not vanish. Then, the matrix $\widetilde{M}(z)$ satisfies the block structure condition of Definition 1.2 on a neighbourhood of $\underline{z}$. 
Proof. The hyperbolicity assumption implies that condition $i$ ) of Assumption 1.4 is satisfied. Consider next $\underline{z} \in \mathcal{U} \times \Sigma_{0}$ and $\underline{\xi} \in \mathbb{R}$ such that $\widetilde{\Delta}(\underline{z}, \underline{\xi})=0$. Note that $(\eta, \xi) \neq 0$ since $(\underline{\tau}, \eta) \neq 0$. Therefore, there is an eigenvalue $\bar{\lambda}$ of $\xi A+\eta B$ such that $\underline{\tau}=-\lambda(\underline{a}, \underline{\eta}, \bar{\xi})$. The constant multiplicity assumption implies that (1.8) is satisfied near $(\underline{z}, \underline{\xi})$.

Consider $(z, \xi)$ in a neighbourhood of $(\underline{z}, \underline{\xi})$. Let $\Pi(a, \eta, \xi)$ denote the eigenprojector associated to $\lambda$, extended to a complex neighbourhood of $\underline{\xi}$. Then

$$
(\xi A+G(z)) \Pi(\xi)=0, \quad \text { when } \tau-i \gamma+\lambda(a, \eta, \xi)=0
$$

Since $\xi A_{b}+G_{b}$ is invertible, the block decomposition (3.5) implies that when $\tau-i \gamma+\lambda(a, \eta, \xi)=0$, the range of $\Pi$ is contained in $\widetilde{\mathbb{K}}(z)$. Therefore, there is a $C^{\infty}$ projector $\widetilde{\Pi}(z, \xi)$ such that

$$
(\xi \widetilde{A}+\widetilde{G}(z)) \widetilde{\Pi}(z, \xi)=0, \quad \text { when } \tau-i \gamma+\lambda(a, \eta, \xi)=0 .
$$

Therefore (1.9) is satisfied and Theorem 3.3 follows from Theorem 1.5.

Remark that Theorem 3.1 follows from Theorem 3.3. When $\operatorname{det} G_{11}(z) \neq 0$, the coefficient of $\xi^{N^{\prime}}$ in $\Delta$ doe not vanish. Therefore $\Delta(\underline{z}, \cdot)=\widetilde{\Delta}(\underline{z}, \cdot)$ and the matrices $M^{\prime}(z)$ and $\widetilde{M}(z)$ are conjugated.

\section{$4 \quad$ Higher order systems}

In this section, we extend Theorem 1.3 to systems of equations of arbitrary order. Consider a system

$$
\begin{aligned}
P(a, \tau, \eta, \xi) & :=I_{N} \tau^{m}+\sum_{j=0}^{m-1} P_{m-j}(a, \eta, \xi) \tau^{j} \\
& =A_{0}(a) \xi^{m}+\sum_{j=0}^{m-1} A_{m-j}(a, \tau, \eta) \xi^{j}
\end{aligned}
$$

where $P_{j}$ [resp. $A_{j}$ ] is a $N \times N$ matrix, $C^{\infty}$ in $a$ and polynomial of degree $j$ in $(\eta, \xi)$ [resp. $(\tau, \eta)]$. We assume here that the boundary is not characteristic, i.e.

$$
\forall a \in \mathcal{U}, \quad \operatorname{det} A_{0}(a) \neq 0 .
$$

In the study of boundary value problems for $P$, one considers $P(a, \tau-i \gamma, \eta, \xi)$ and the associated first order pseudodifferential $\mathrm{Nm} \times \mathrm{Nm}$ system of symbol

$$
L(z, \xi)=\xi I_{N m}+\widetilde{M}(z)
$$

where

$$
\widetilde{M}(z)=\left[\begin{array}{cccc}
0 & -\Lambda & \ldots & 0 \\
\vdots & \ddots & \ddots & \vdots \\
0 & \ldots & 0 & -\Lambda \\
M_{m} & \cdots & & M_{1}
\end{array}\right]
$$


where $M_{j}=\Lambda^{1-j} A_{0}^{-1} A_{j}$ and $\Lambda=\left(\tau^{2}+|\eta|^{2}+\gamma^{2}\right)^{-1 / 2} I_{N}$ (see e.g. [1]). In this reduction the matrix $\widetilde{M}$ is homogeneous of degree one in $(\tau, \eta, \gamma)$.

The characteristic determinant is

$$
\Delta(a, \tau, \eta, \xi)=\operatorname{det} P(a, \tau, \eta, \xi)=\operatorname{det} A_{0}(a) \operatorname{det}\left(\xi I_{N m}+\widetilde{M}(z)\right)
$$

Definition 4.1. $P$ is a symmetrizable hyperbolic system of constant multiplicity if

i) for all $a \in \mathcal{U}$ and $(\eta, \xi) \in \mathbb{R}^{d} \backslash\{0\}$, all the roots in $\tau$ of $\Delta$ are real,

ii) locally their multiplicity is constant,

iii) the dimension of $\operatorname{ker} P(a, \tau, \eta, \xi)$ is equal to the multiplicity of $\tau$ as a root of $\Delta$.

Theorem 4.2. If $P$ is symmetrizable hyperbolic system with constant multiplicity, then the matrix $\widetilde{M}(z)$ defined in (4.3) satisfies the block structure condition.

Proof. The hyperbolicity assumption implies that condition $i$ ) of Assumption 1.4. is satisfied. Consider the $\mathrm{Nm} \times \mathrm{Nm}$ matrix

$$
\widetilde{P}(a, \eta, \xi)=\left[\begin{array}{cccc}
0 & -I_{N} & \cdots & 0 \\
\vdots & \ddots & \ddots & \vdots \\
0 & \cdots & 0 & -I_{N} \\
P_{m} & \cdots & & P_{1}
\end{array}\right]
$$

Then

$$
\operatorname{det}\left(\tau I_{N m}+\widetilde{P}(a, \eta, \xi)\right)=\Delta(a, \tau, \eta, \xi) .
$$

Moreover, if $-\tau$ is an eigenvalue of $\widetilde{P}(a, \eta, \xi)$, the associated eigenspace is $J_{\tau}$ ker $P(a, \tau, \eta, \xi)$ where $J_{\tau}$ is the mapping from $\mathbb{C}^{N}$ into $\mathbb{C}^{N m}$ :

$$
J_{\tau}: u \mapsto\left(u, \tau u, \ldots, \tau^{m-1} u\right)
$$

In particular,

$$
\operatorname{dim} \operatorname{ker} P(a, \tau, \eta, \xi)=\operatorname{dim} \operatorname{ker}\left(\tau I_{N m}+\widetilde{P}(a, \eta, \xi)\right)
$$

Thus, for $(\eta, \xi) \neq 0$, the eigenvalues $\lambda_{j}(a, \eta, \xi)$ of $\widetilde{P}$ are real, semi-simple and have constant multiplicity. Moreover, the eigenprojectors are of the form

$$
\Pi_{j}^{\sharp}\left(u_{1}, \ldots, u_{m}\right)=J_{-\lambda_{j}} \Pi_{j} u_{1}
$$

where $\Pi_{j}=\Pi_{j}(a, \eta, \xi)$ is a projector onto $\operatorname{ker} P\left(a,-\lambda_{j}, \eta, \xi\right)$. Since $\Pi_{j}^{\sharp}$ is smooth, $\Pi_{j}$ is a $C^{\infty}$ function of $(a, \eta, \xi)$. In addition, the eigenvalues $\lambda_{j}$, the eigenprojectors $\Pi_{j}^{\sharp}$ and hence $\Pi_{j}$ extend holomorphically in $\xi$.

Consider $z \in \mathcal{U} \times \Sigma_{0}$ and $\underline{\xi} \in \mathbb{R}$ such that $\Delta(\underline{z}, \underline{\xi})=0$. By homogeneity, $(\underline{\eta}, \underline{\xi}) \neq 0$ and there is $j$ such that $\underline{\tau}+\lambda_{j}(\underline{a}, \underline{\eta}, \underline{\xi})=0$. The constant multiplicity assumption and (4.5) (4.7), imply that the condition (1.8) is satisfied, with $\alpha$ equal to the multiplicity of $\lambda_{j}$. Next, we introduce the mapping

$$
J_{z, \xi}: u \mapsto\left(u, \xi \Lambda(z)^{-1} u, \ldots, \xi^{m-1} \Lambda(z)^{1-m} u\right)
$$


and we remark that when $\xi$ is close to $\underline{\xi}$ and $\tau-i \gamma+\lambda_{j}(a, \eta, \xi)=0$,

$$
\widetilde{\Pi}_{j}(a, \eta, \xi)\left(u_{1}, \ldots, u_{m}\right)=\widetilde{J}_{z, \xi} \Pi_{j}(a, \eta, \xi) u_{1}
$$

is a projector of rank $\alpha$ onto onto the kernel of $\xi I_{N m}+M(z)$. Therefore the property (1.9) is satisfied and Theorem 4.3 follows from Theorem 1.5.

\section{$5 \quad$ Examples}

5.1. Consider the linearized equations of isentropic gas dynamics in space dimension three

$$
\left\{\begin{array}{l}
X \dot{\rho}+\rho \operatorname{div} \dot{v}=f, \\
X \dot{v}_{j}+\rho^{-1} c^{2} \partial_{x_{j}} \dot{\rho}=g_{j} \quad \text { for } \quad 1 \leq j \leq 3,
\end{array}\right.
$$

where $X=\partial_{t}+v_{1} \partial_{x_{1}}+v_{2} \partial_{x_{2}}+v_{3} \partial_{x_{3}}$. The unknowns are $(\dot{\rho}, \dot{v})$. The symbol of (5.1) is of the form (1.1) with parameters $a=(\rho, c, v)$. We choose $\left\{x_{1}=0\right\}$ as a boundary. It is non characteristic when $v_{1} \notin\{0,-c, c\}$. The characteristic polynomial is

$$
\Delta(a, \tau, \xi)=(\tau+v \cdot \xi)^{2}\left((\tau+v \cdot \xi)^{2}-c^{2}|\xi|^{2}\right) .
$$

It is of degree 4 in $\xi_{1}$.

The blocks $M_{k}$ which correspond to roots of $\left.(\tau+v \cdot \xi)^{2}-c^{2}|\xi|^{2}\right)$ are studied exactly as in the strictly hyperbolic case. On the other hand, the root in $\xi_{1}$ of $\tau+v \cdot \xi$ is always simple and the corresponding block $M_{k}$ is diagonal.

The equation (5.1) occurs in the study of shock fronts for Euler's equation ([3]) and also in the study of shallow water waves ([5]).

5.2. The equations of linear elasticity read

$$
P\left(\partial_{t}, \partial_{y}, \partial_{x}\right) u:=\partial_{t}^{2} u-(\lambda+\mu) \operatorname{grad} \operatorname{div} u-\mu \Delta u=f .
$$

In space dimension $d$, this is a $d \times d$ systems of second order equations. The characteristic equation is

$$
\left(\tau^{2}-\mu|\xi|^{2}\right)^{d-1}\left(\tau^{2}-(\lambda+2 \mu)|\xi|^{2}\right)
$$

The eigenvalues $\pm \sqrt{\mu}|\xi|$ [resp. $\pm \sqrt{\lambda+2 \mu}|\xi|]$ have constant multiplicity equal to $d-1$ [resp. 1 ] and the dimension of ker $P(\tau, \eta, \xi)$ is equal to the multiplicity. Therefore, Theorem 4.2 applies and the block structure condition is satisfied. Here both factors $\left(\tau^{2}-c|\xi|^{2}\right)$ have multiple roots in $\xi_{1}$ (glancing modes) when $\tau^{2}=c|\eta|^{2}$. 
5.3 The three dimensional Maxwell's equations in vacuum read

$$
\left\{\begin{array}{l}
\partial_{t} E-\operatorname{curl} B=0 \\
\partial_{t} B+\operatorname{curl} E=0
\end{array}\right.
$$

Then

$$
\Delta(\tau, \xi)=\tau^{2}\left(\tau^{2}-|\xi|^{2}\right)^{2}
$$

The system is symmetric hyperbolic and the multiplicities are constant. The boundary $\left\{x_{1}=0\right\}$ is characteristic and the condition (3.1) is satisfied. When $\underline{\tau}-i \underline{\gamma} \neq 0, M^{\prime}(z)$ is a $4 \times 4$ matrix and Theorem 3.1 applies (see also [5]). Note that the characteristic polynomial of $M^{\prime}(z)$ is $\left(\xi^{2}-(\tau-i \gamma)^{2}+|\eta|^{2}\right)^{2}$. In this example, real roots in $\xi$ occur only when $\gamma=0$ and $\tau^{2} \geq|\eta|^{2}$, in which case, with the notations of $\S 3, \operatorname{det} G_{11} \neq 0$. In the block decomposition of $M^{\prime}$, double Jordan blocks occur when $\underline{\tau}^{2}=|\underline{\eta}|^{2}$.

\section{References}

[1] J.ChazArain - A.Piriou, Introduction à la théorie des équations aux dérivées partielles linéaires, Gauthier-Villars, Paris 1981 \& Introduction to the Theory of Linear Partial Differential equations, North Holland, Amsterdam, 1982

[2] H.O. KReIss, Initial Boundary Value Problem for Hyperbolic Systems, Comm. on Pure and Appl. Math., 23 (1970), pp 277-298.

[3] A. Majda, The Stability of Multidimensional Shocks, Memoirs of the Amer.Math.Soc. 275 (1983).

[4] A. MajdA, The Existence of Multidimensional Shocks, Memoirs of the Amer.Math.Soc. 281 (1983).

[5] A.Majda, S.Osher, Initial boundary value problems for hyperbolic equations with uniformly characteristic boundary, Comm. Pure Appl. Math., 28 (1975), pp 607-676.

[6] G.MÉtivier, Stability of Multidimensional Shocks, Lecture notes, Kochel summer school, 1999, to appear in Recent Advances in the Theory of Shock Waves, Progress in Nonlinear Diff.Equ., Birkäuser.

[7] A.Mokrane, Problèmes mixtes hyperboliques non linéaires, Thèse, Université de Rennes 1, 1987.

[8] J.Ralston, Note on a Paper of Kreiss, Comm.Pure and Appl.Math., 24 (1971) pp 759-762. 